(C) The Authors 2018. This is an Open Access article, distributed under the terms of the Creative Commons

Attribution licence (http://creativecommons.org/licenses/by/4.0/), which permits unrestricted re-use,

distribution, and reproduction in any medium, provided the original work is properly cited.

\title{
Adherence to the Mediterranean diet and risk of lung cancer in the Netherlands Cohort Study
}

\author{
Maya Schulpen ${ }^{1 *}$ and Piet A. van den Brandt ${ }^{1,2}$ \\ ${ }^{1}$ Department of Epidemiology, GROW - School for Oncology and Developmental Biology, Maastricht University Medical \\ Centre, PO Box 616, 6200 MD Maastricht, the Netherlands \\ ${ }^{2}$ Department of Epidemiology, CAPHRI - School for Public Health and Primary Care, Maastricht University Medical Centre, \\ PO Box 616, 6200 MD Maastricht, the Netherlands
}

(Submitted 25 April 2017 - Final revision received 21 November 2017 - Accepted 12 December 2017)

\section{Abstract}

The evidence on a cancer-protective effect of the Mediterranean diet (MD) is still limited. Therefore, we investigated the association between MD adherence and lung cancer risk. Data were used from 120852 participants of the Netherlands Cohort Study (NLCS), aged 55-69 years. Dietary habits were assessed at baseline (1986) using a validated FFQ and alternate and modified Mediterranean diet scores (aMED and mMED, respectively), including and excluding alcohol, were calculated. After 20.3 years of follow-up, 2861 lung cancer cases and 3720 subcohort members (case-cohort design) could be included in multivariable Cox regression analyses. High (6-8) $v$. low (0-3) aMED excluding alcohol was associated with non-significantly reduced lung cancer risks in men and women with hazard ratios of 0.91 (95\% CI $0 \cdot 72,1 \cdot 15)$ and 0.73 (95\% CI 0.49, 1.09), respectively. aMED-containing models generally fitted better than mMED-containing models. In never smokers, a borderline significant decreasing trend in lung cancer risk was observed with increasing aMED excluding alcohol. Analyses stratified by the histological lung cancer subtypes did not identify subtypes with a particularly strong inverse relation with MD adherence. Generally, the performance of aMED and World Cancer Research Fund/American Institute for Cancer Research dietary score variants without alcohol was comparable. In conclusion, MD adherence was non-significantly inversely associated with lung cancer risk in the NLCS. Future studies should focus on differences in associations across the sexes and histological subtypes. Furthermore, exclusion of alcohol from MD scores should be investigated more extensively, primarily with respect to a potential role of the MD in cancer prevention.

Key words: Mediterranean diet: Lung cancer: Subtypes: Cohort studies: Epidemiology

Lung cancer was the leading cause of cancer death in the world in 2012. In that year, 1.8 million people were diagnosed, making lung cancer the most frequently diagnosed cancer in men and the third most common cancer in women worldwide ${ }^{(1)}$. The prognosis of lung cancer is poor, with 5-year survival rates of $19 \%$ in the $\mathrm{USA}^{(2)}$ and $17 \%$ in the Netherlands ${ }^{(3)}$ for lung cancers diagnosed around 2010. Tobacco smoking is the primary risk factor, but diet may influence lung cancer risk as well ${ }^{(4)}$.

The traditional Mediterranean diet (MD) is a plant-based diet. Vegetables, legumes, fruits, nuts, whole grains and fish are consumed in large amounts in this dietary pattern, whereas consumption of red and processed meats, high-fat dairy products, refined grains and sweets is limited. Olive oil is the principal source of fat resulting in a relatively high ratio of MUFA: SFA. A final characteristic of this diet is the moderate consumption of red wine, mainly during meals ${ }^{(5-7)}$. Higher MD adherence has been associated with numerous health benefits including reduced all-cause mortality, and decreased incidence of and mortality from $\mathrm{CVD}^{(8-10)}$. Recently, higher MD adherence has been associated with a reduced lung cancer risk in three different cohort studies ${ }^{(11-13)}$. However, the number of lung cancer cases was small in two of the three studies, and sex-specific associations and possible differences in associations of MD adherence with the four major histological lung cancer subtypes were not investigated elaborately. Even though the association between alcohol consumption and lung cancer risk is still unclear, these previous studies included alcohol in the MD score used ${ }^{(14)}$.

The present study was conducted among men and women between the ages of 55 and 69 years, who participated in the Netherlands Cohort Study (NLCS). In this paper, we aimed to

Abbreviations: AIC, Akaike's information criterion; aMED, alternate Mediterranean diet score; aMEDr, alternate Mediterranean diet score without the alcohol component; HR, hazard ratio; MD, Mediterranean diet; mMED, modified Mediterranean diet score; mMEDr, modified Mediterranean diet score without the alcohol component; NIH-AARP, National Institutes of Health-American Association of Retired Persons; NLCS, Netherlands Cohort Study; WCRF/AICR, World Cancer Research Fund/American Institute for Cancer Research.

* Corresponding author: M. Schulpen, fax +31 43 3884128, email maya.schulpen@maastrichtuniversity.nl 
investigate the association between MD adherence and the risk of lung cancer. Age-specific incidence rates of lung cancer in the Netherlands in the study period (1986-2006) were 281/100 000 for people aged between 60 and 74 years in 1990 and 318/100 000 for people aged 75 years or older in $2005^{(3)}$. In addition, we tested the heterogeneity of the association of MD adherence with lung cancer risk between the sexes and four major histological lung cancer subtypes, and evaluated the effect of exclusion of alcohol from the MD scores.

\section{Methods}

\section{Study design and lung cancer follow-up}

The NLCS is a prospective population-based cohort study that was initiated in September 1986 and has been described previously $^{(15-18)}$. At enrolment, 58279 men and 62573 women, aged between 55 and 69 years, filled out a self-administered questionnaire regarding their dietary habits and other cancer risk factors. The NLCS was approved by the institutional review boards from Maastricht University and the Netherlands Organization for Applied Scientific Research. To allow efficient data processing, the nested case-cohort approach was applied, in which the number of person-years at risk is estimated based on a subcohort ( $n$ 5000) that was randomly sampled just after baseline. Vital status of subcohort members was biennially verified $^{(15,18)}$. Incident cancers in the NLCS cohort were detected by annual computerised record linkage with the Netherlands Cancer Registry and the Dutch Pathology Registry ('Pathologisch-Anatomisch Landelijk Geautomatiseerd Archief', PALGA) ${ }^{(16)}$. Reported computer matches, based on a linkage key, were estimated to have a sensitivity and positive predictive value of $98 \%{ }^{(16)}$. Computerised linkage was followed by visual inspection in order to distinguish true matches from false positives, which maximised the positive predictive value to $100 \%$, whereas remaining the sensitivity unaltered ${ }^{(16)}$. It was estimated that the completeness of follow-up through record linkage with the Netherlands Cancer Registry and PALGA exceeded $95 \%{ }^{(19)}$. Incident lung cancers (International Classification of Diseases for Oncology, code C34, 3rd ed.) were detected until $20 \cdot 3$ years of follow-up. Cases with lung cancers other than carcinoma, in situ lung carcinoma or not microscopically confirmed lung cancer were excluded. Cases and subcohort members with prevalent cancer at baseline (except skin cancer), and incomplete or inconsistent (dietary) questionnaires were not eligible as well. In the end, 4084 subcohort members and 3261 lung cancer cases were eligible for inclusion in the analyses. Of the eligible study population, 2861 lung cancer cases and 3720 subcohort members were included in the Cox proportional hazards models as a result of missing values in covariates (Fig. 1).

\section{Exposure assessment}

At baseline, NLCS participants completed a self-administered, 150-item, semi-quantitative FFQ measuring dietary habits over the preceding year, which was validated by 9-d dietary records filled out over three different seasons ${ }^{(17)}$. In addition, reproducibility of the single baseline measurement of the FFQ was investigated by means of five annually repeated measurements of dietary intake in independent random samples from the subcohort. Considering all nutrients, the average test-retest correlation of the FFQ was 0.66. Over 5 years, the correlation between the baseline and repeated measurement of the FFQ had declined on average 0.07 , suggesting that the FFQ was able to rank subjects according to their nutrient intake relatively well

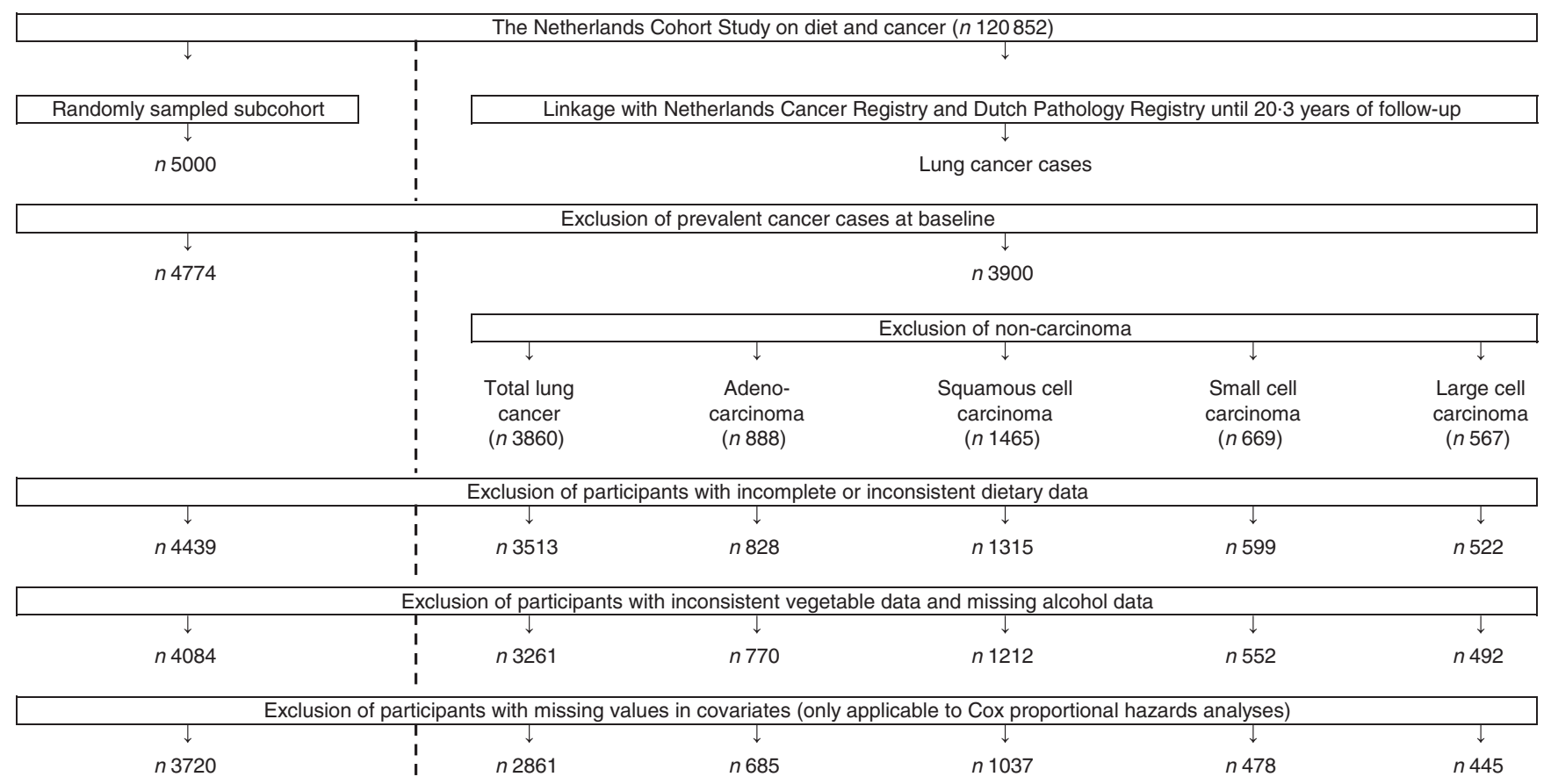

Fig. 1. Flow diagram of the number of subcohort members and lung cancer cases who were included in the analyses (case-cohort design). 
over at least a 5 -year period ${ }^{(20)}$. In order to derive mean daily nutrient intakes from the questionnaire data, the 1986 Dutch food composition (NEVO) table was utilised ${ }^{(21)}$.

\section{Mediterranean diet adherence}

The alternate and modified Mediterranean diet scores (aMED and $\mathrm{mMED}$, respectively), which were both derived from the traditional Mediterranean diet score (tMED) developed in 1995 by Trichopoulou et al., were applied to measure relative MD adherence $^{(22-26)}$. Before calculation of the aMED and MMED, dietary intakes were standardised to a total daily energy intake of $8368 \mathrm{~kJ}$ (2000 kcal) for women and $10460 \mathrm{~kJ}$ (2500 kcal) for men $^{(22,26)}$.

aMED considers the intake of nine food components, which are scored as either 0 or 1 using population-based cut-offs, resulting in a sum score varying from 0 (minimal adherence) to 9 (maximal adherence) $^{(25,26)}$. A score of 1 is assigned to daily intakes at or above the sex-specific median for components presumed to be beneficial (vegetables (excluding potatoes), legumes, fruits, nuts, whole grains, fish and MUFA:SFA ratio), whereas the intake of red and processed meats is scored inversely. Finally, moderate alcohol consumption $(5-25 \mathrm{~g} / \mathrm{d}$ for both men and women) is assigned 1 point as well ${ }^{(25,26)}$.

$\mathrm{mMED}^{(24)}$, developed for usage in non-Mediterranean populations, differs from aMED with regard to the food components included. In mMED, fruits and nuts are considered as one component, the cereal component includes whole and refined grains, total meat consumption is scored, dairy consumption is included as component, and the unsaturated (MUFA + PUFA) fatty acid:SFA ratio is used. Also, the definition of moderate alcohol consumption is different (men: $10-50 \mathrm{~g} / \mathrm{d}$; women: $5-25 \mathrm{~g} / \mathrm{d})^{(24)}$.

Because of the unclear association between alcohol intake and lung cancer risk $^{(14)}$ and variability in the definition of moderate alcohol consumption, aMED and mMED variants without alcohol were created, resulting in sum scores ranging from 0 to 8 points. We refer to these reduced scores as alternate Mediterranean diet score without the alcohol component (aMEDr) and modified Mediterranean diet score without the alcohol component (mMEDr), respectively.

\section{Statistical analyses}

Initially, all analyses were performed separately for men and women. Descriptive statistics were used to assess baseline characteristics of the subcohort.

The relation between MD adherence and lung cancer incidence was evaluated by running Cox proportional hazards models with follow-up as the timing variable to estimate hazard ratios (HR) and $95 \%$ CI. Person-years at risk of subcohort members were calculated from baseline until lung cancer diagnosis, death, emigration, loss to follow-up or end of follow-up, whichever occurred first. Because the variance is increased in a case-cohort design, standard errors were calculated using the Huber-White sandwich estimator ${ }^{(27)}$. Scaled Schoenfeld residuals tests, smoothed scaled Schoenfeld residuals plots and $-\ln$ $(-\ln )$ survival plots confirmed that the proportional hazards assumption was met ${ }^{(28)}$. aMEDr and mMEDr were included in the analyses as categorical (low: 0-3; middle: 4-5; high: 6-8 points) and continuous (per 2-point increment) variables. For each exposure, three models were run, varying with respect to the covariates (literature-based) included. The age-adjusted analyses were adjusted for age at baseline (years) and sex (except sex-specific models). Additional adjustment for cigarette smoking status (never, former, current), duration (years, centred) and frequency (cigarettes smoked per day, centred) was applied in the smoking-adjusted models. The fully adjusted analyses were also adjusted for daily energy intake (kJ (kcal)), alcohol consumption $(0,>0-<5, \geq 5-<15, \geq 15-<30, \geq 30 \mathrm{~g} / \mathrm{d})$, BMI $\left(<18 \cdot 5, \geq 18 \cdot 5-<25, \quad \geq 25-<30, \quad \geq 30 \mathrm{~kg} / \mathrm{m}^{2}\right)$, nonoccupational physical activity $(\leq 30,>30-\leq 60,>60-\leq 90$, $>90 \mathrm{~min} / \mathrm{d}$ ), highest level of education (primary school or lower vocational, secondary school or medium vocational, higher vocational or university), family history of lung cancer (no, yes) and history of physician-diagnosed chronic bronchitis (no, yes). Tests for trends were performed by considering the categorical MD score variables as continuous parameters.

Performances of models with aMEDr and mMEDr were compared using Akaike's information criterion (AIC) ${ }^{(29)}$. In addition, AIC was used to compare the fit of models containing aMEDr and mMEDr, to the fit of models containing the original aMED and mMED including alcohol (categorised: 0-3 (low), 4-5 (middle) and 6-9 (high); continuous: per 2-point increment). The latter models did not include alcohol consumption as covariate. Based on these analyses, it was decided to assess MD adherence in the remainder of the analyses by aMEDr. Population attributable fractions (PAF) were calculated using the STATA command punafcc, to determine the proportion of lung cancers that could be prevented if everyone had high MD adherence ${ }^{(30,31)}$. The effects of an alternative scenario, in which subjects with low and middle MD adherence moved one category upwards, were evaluated by means of preventable proportions ${ }^{(32)}$

The relation between MD adherence and lung cancer was also evaluated separately for the four major histological lung cancer subtypes: adenocarcinoma, squamous cell carcinoma, small cell carcinoma and large cell carcinoma. Heterogeneity across the subtypes was tested using a competing risks procedure. Standard errors of the difference were estimated using a bootstrapping method, especially developed for the case-cohort design, to prevent overestimation due to the absence of independence $^{(33,34)}$.

Potential effect-modification was evaluated by conducting Wald tests on interaction terms between the exposure of interest and age at baseline, smoking status, BMI, non-occupational physical activity, alcohol consumption and family history of lung cancer. In addition, analyses were performed within strata of the same variables to illustrate potential modifications and to evaluate potential residual confounding by these lung cancer risk factors. Sensitivity analyses were conducted by dividing the follow-up in three periods ( $\leq 2$ years, $>2-\leq 10$ years, $>10$ years) and excluding cases diagnosed during the first 2 years of follow-up. Moreover, the potential impact of unmeasured confounders was evaluated using rule-out approach sensitivity analysis as proposed by Schneeweiss ${ }^{(35)}$. 
The contribution of the individual aMEDr components to the observed associations was investigated by alternately deleting each component from the sum score using the methods described by Trichopoulou et al. ${ }^{(36)}$. Because this procedure reduces the maximum score, we multiplied the regression coefficients per 2-point increment by 8/9 before exponentiating them, to obtain HR that could be compared with effect estimates of the original score. Furthermore, the change in beneficial effect after deletion of one component was divided by the beneficial effect of the original score to acquire the percentage reduction of the inverse association. Analyses were adjusted by the subtracted component and all covariates included in the fully adjusted model.

Finally, the population-dependent assignment of scores is a potential drawback of the MD scores used, especially in nonMediterranean populations. Therefore, we compared the performances of models containing the relative aMED variants and models containing absolute scores based on the dietary part of the recommendations to prevent cancer of the World Cancer Research Fund/American Institute for Cancer Research (WCRF/ AICR) with and without alcohol ${ }^{(4)}$. We operationalised the recommendations concerning consumption of foods and drinks that promote weight gain, plant foods, red and processed meats, alcohol and salt. The absolute cut-offs used per (sub) recommendation as well as the calculation of the score have been described in detail in a previous NLCS article ${ }^{(37)}$ and were based on the methods used in the EPIC cohort ${ }^{(38,39)}$. This resulted in a score ranging from 0 to 5 points with higher scores reflecting higher adherence to the WCRF/AICR recommendations. Analogous to aMED, we also created a WCRF/AICR score excluding the alcohol recommendation, which had a maximum value of 4 points. The continuous WCRF/AICR score variables were included in fully adjusted Cox proportional hazards models to estimate HR and 95\% CI per SD increment (sex specific). A similar approach was applied to aMED and aMEDr and the performances of models including WCRF/AICR score variables and aMED variables were compared using AIC. Analyses were carried out using STATA14 (StataCorp LP) and Microsoft Excel 2010 (Microsoft Corporation). $P$ values below 0.05 were considered to be statistically significant.

\section{Results}

After 20.3 years of follow-up, 3261 (men: 2777; women: 484) of the detected lung cancer cases met the eligibility criteria and were included in the present study. Squamous cell carcinoma (39.5\%) was the most frequently diagnosed subtype in men, followed by adenocarcinoma (21.8\%), small cell carcinoma (16.7\%) and large cell carcinoma (15.0\%). In women, these subtypes comprised $23 \cdot 6,33 \cdot 9,18 \cdot 2$ and $15 \cdot 5 \%$, respectively. The remainder of the carcinomas detected was categorised as unspecified (6.9\% in men and $8.9 \%$ in women).

Subcohort members (50.4\% male sex) had a median age of 61 years at baseline and the majority $(64.9 \%)$ was a former or current smoker. Mean values of aMEDr and mMEDr were approximately 4 in both male and female subcohort members. Sex-specific baseline characteristics of subcohort members are presented by aMEDr and mMEDr category in Table 1. Subcohort members with higher MD adherence were more often highly educated, more physically active and less often current smokers. Lower mean daily energy intakes were reported in both men (aMEDr and mMEDr) and women (mMEDr) with higher MD adherence. Furthermore, inverse associations with MD conformity in men were observed for alcohol consumption and having a lung cancer family history. In contrast, women with higher MD adherence were younger, consumed more alcohol (aMEDr), more often had a positive lung cancer family history (mMEDr) and less often had suffered from chronic bronchitis (aMEDr).

Because of missing values in covariates included in the multivariable analyses, 2861 lung cancer cases (men: 2413; women: 448) and 3720 subcohort members (men: 1834; women: 1886) were included in the Cox models. Sex-specific HR and 95\% CI for the association between MD adherence, measured by various MD scores, and lung cancer risk are displayed in Table 2. Statistically significant inverse associations of MD adherence with lung cancer risk were observed in all ageadjusted analyses in both sexes. After additional correction for cigarette smoking (cigarette smoking status, duration and frequency), the associations attenuated and were no longer statistically significant, but generally remained inverse. However, the association disappeared in men when conformity to MD was assessed using mMEDr. Additional adjustment for other potential confounders did not result in noticeable changes in the estimates. The inverse association between aMEDr and lung cancer seemed to be slightly stronger in women compared with men. However, this heterogeneity between the sexes was not statistically significant. Fully adjusted HR for the middle and high adherence categories, respectively, were 0.87 (95\% CI $0.65,1.15)$ and 0.73 (95\% CI 0.49, 1.09) in women, and 0.86 (95\% CI $0.73,1.02)$ and 0.91 (95\% CI $0.72,1.15)$ in men, using the low category as reference. Judged by AIC, models containing aMEDr had a better fit than mMEDr-containing models. Furthermore, model fits were worse when alcohol consumption was included in the score. Therefore, as was already mentioned in the Methods section, aMEDr was used to assess MD adherence in the remainder of the analyses. Assuming causality, PAF showed that $18.9 \%(95 \% \mathrm{CI}-12 \cdot 6,41 \cdot 6)$ and $2.0 \%(95 \% \mathrm{CI}$ $-19 \cdot 1,19 \cdot 4)$ of the lung cancers in women and men, respectively, would be prevented if the entire population had high MD adherence according to aMEDr. A more realistic scenario in which subjects with low and middle aMEDr values moved one category upwards would prevent $12 \cdot 1 \%$ of the female and $4.0 \%$ of the male lung cancer cases, respectively.

Table 3 displays the results of subtype-specific Cox regression analyses. Although there seemed to be some variations in the strength of the associations across the subtypes, particularly in men, heterogeneity tests were not statistically significant. In men, aMEDr appeared to be non-significantly inversely associated with small cell carcinoma risk, but not with adenocarcinoma risk. Middle MD adherence was associated with (borderline) significantly reduced risks of squamous and large cell carcinoma in men. In women, higher aMEDr values were associated with non-significantly reduced risks of adenocarcinoma, squamous cell carcinoma and small cell carcinoma. 
Table 1. Sex-specific baseline characteristics of the subcohort by alternate Mediterranean diet score without the alcohol component (aMEDr) and modified Mediterranean diet score without the alcohol component (mMEDr) category*

(Mean values and standard deviations; medians and interquartile ranges (IQR))

\begin{tabular}{|c|c|c|c|c|c|c|}
\hline & \multicolumn{3}{|c|}{ aMEDr } & \multicolumn{3}{|c|}{ mMEDr } \\
\hline & $0-3$ & $4-5$ & $6-8$ & $0-3$ & $4-5$ & $6-8$ \\
\hline \multicolumn{7}{|l|}{ Men } \\
\hline$n$ & 855 & 887 & 315 & 777 & 931 & 349 \\
\hline \multicolumn{7}{|l|}{ Age (years) $\dagger$} \\
\hline Median & 61 & 61 & 61 & 61 & 61 & 62 \\
\hline IQR & 7 & 7 & 7 & 7 & 7 & 7 \\
\hline Never cigarette smokers (\%) & 11.5 & $13 \cdot 1$ & $15 \cdot 9$ & $11 \cdot 7$ & $14 \cdot 7$ & $10 \cdot 3$ \\
\hline Former cigarette smokers (\%) & 48.0 & $52 \cdot 4$ & $62 \cdot 2$ & $48 \cdot 3$ & $51 \cdot 7$ & 61.6 \\
\hline Higher vocational education or university (\%) & $16 \cdot 0$ & $20 \cdot 6$ & $24 \cdot 6$ & $18 \cdot 1$ & $20 \cdot 2$ & $19 \cdot 7$ \\
\hline Family history of lung cancer (\%) & 9.7 & $9 \cdot 4$ & $9 \cdot 2$ & $10 \cdot 9$ & 8.6 & $8 \cdot 6$ \\
\hline \multicolumn{7}{|l|}{ Alcohol consumption $(\mathrm{g} / \mathrm{d}) \dagger$} \\
\hline Median & 9.8 & $10 \cdot 4$ & 8.5 & 11.0 & $9 \cdot 6$ & 8.6 \\
\hline IQR & $21 \cdot 1$ & $21 \cdot 7$ & $17 \cdot 6$ & $21 \cdot 8$ & $20 \cdot 2$ & $18 \cdot 3$ \\
\hline \multicolumn{7}{|l|}{ Daily energy intake (kJ) } \\
\hline Mean & 9100 & 9146 & 8619 & 9272 & 9004 & 8657 \\
\hline SD & 2138 & 2138 & 1778 & 2121 & 2063 & 2063 \\
\hline \multicolumn{7}{|l|}{ Daily energy intake (kcal) } \\
\hline Mean & 2175 & 2186 & 2060 & 2216 & 2152 & 2069 \\
\hline SD & 511 & 511 & 425 & 507 & 493 & 493 \\
\hline \multicolumn{7}{|l|}{ BMI $\left(\mathrm{kg} / \mathrm{m}^{2}\right)$} \\
\hline Mean & $25 \cdot 0$ & $25 \cdot 0$ & 24.7 & 24.9 & 24.9 & $25 \cdot 0$ \\
\hline SD & $2 \cdot 6$ & $2 \cdot 6$ & 2.5 & $2 \cdot 6$ & $2 \cdot 5$ & $2 \cdot 7$ \\
\hline \multicolumn{7}{|l|}{ Non-occupational physical activity $(\min / \mathrm{d}) \dagger$} \\
\hline Median & $55 \cdot 7$ & $64 \cdot 3$ & $70 \cdot 7$ & $55 \cdot 7$ & 68.6 & $64 \cdot 3$ \\
\hline IQR & $60 \cdot 0$ & $64 \cdot 3$ & 61.4 & $60 \cdot 0$ & 62.9 & $64 \cdot 3$ \\
\hline History of chronic bronchitis (\%) & 8.5 & $6 \cdot 8$ & $7 \cdot 3$ & 8.5 & $6 \cdot 2$ & $9 \cdot 2$ \\
\hline \multicolumn{7}{|l|}{ Women } \\
\hline \multirow{2}{*}{\multicolumn{7}{|c|}{ Age (years) $\dagger$}} \\
\hline & & & & & & \\
\hline Median & 62 & 61 & 60 & 62 & 61 & 61 \\
\hline IQR & 7 & 7 & 7 & 7 & 7 & 6 \\
\hline Never cigarette smokers (\%) & $56 \cdot 2$ & $58 \cdot 6$ & $58 \cdot 8$ & $55 \cdot 2$ & $59 \cdot 4$ & $58 \cdot 2$ \\
\hline Former cigarette smokers (\%) & $17 \cdot 7$ & $21 \cdot 6$ & $26 \cdot 6$ & 19.7 & 20.5 & $25 \cdot 6$ \\
\hline Higher vocational education or university (\%) & $7 \cdot 0$ & $10 \cdot 7$ & $12 \cdot 1$ & $9 \cdot 3$ & 8.8 & $12 \cdot 3$ \\
\hline Family history of lung cancer (\%) & $9 \cdot 1$ & 11.5 & 9.5 & 9.2 & $10 \cdot 6$ & $11 \cdot 7$ \\
\hline \multicolumn{7}{|l|}{ Alcohol consumption $(\mathrm{g} / \mathrm{d}) \dagger$} \\
\hline Median & 0.9 & 1.7 & 3.0 & 1.6 & 1.6 & 1.8 \\
\hline IQR & 5.9 & 8.9 & 8.8 & 8.7 & 7.8 & $6 \cdot 2$ \\
\hline \multicolumn{7}{|l|}{ Daily energy intake $(\mathrm{kJ})$} \\
\hline Mean & 7096 & 7012 & 7105 & 7150 & 7037 & 6920 \\
\hline SD & 1640 & 1636 & 1661 & 1607 & 1665 & 1640 \\
\hline \multicolumn{7}{|l|}{ Daily energy intake (kcal) } \\
\hline Mean & 1696 & 1676 & 1698 & 1709 & 1682 & 1654 \\
\hline SD & 392 & 391 & 397 & 384 & 398 & 392 \\
\hline \multicolumn{7}{|l|}{$\mathrm{BMI}\left(\mathrm{kg} / \mathrm{m}^{2}\right)$} \\
\hline Mean & $25 \cdot 1$ & $25 \cdot 1$ & 24.7 & 24.9 & $25 \cdot 1$ & 24.9 \\
\hline SD & 3.6 & $3 \cdot 6$ & 3.1 & 3.6 & 3.5 & 3.5 \\
\hline \multicolumn{7}{|l|}{ Non-occupational physical activity $(\mathrm{min} / \mathrm{d}) \dagger$} \\
\hline Median & $48 \cdot 6$ & 55.7 & 63.6 & $47 \cdot 1$ & $57 \cdot 1$ & $55 \cdot 7$ \\
\hline IQR & $53 \cdot 6$ & 51.4 & 57.9 & 48.9 & 51.4 & 57.9 \\
\hline History of chronic bronchitis (\%) & $5 \cdot 6$ & $5 \cdot 2$ & $4 \cdot 8$ & $5 \cdot 9$ & $4 \cdot 4$ & $6 \cdot 7$ \\
\hline
\end{tabular}

* The $\%$ missing values was $<5 \%$ for all variables included in this table.

$\dagger$ Non-normal distribution of this variable.

Both sexes were combined in the subgroup analyses (online Supplementary Table S1) to increase the power, as there was no evidence of heterogeneity. Stratification by smoking status showed a borderline statistically significant decreasing trend in lung cancer risk with increasing aMEDr in never smokers $(P=0.07)$, but not in former $(P=0.38)$ or current smokers $(P=0 \cdot 28)$. However, the interaction test was not statistically significant $\left(P_{\text {heterogeneity }}=0 \cdot 07\right)$. The inverse association between MD adherence and lung cancer risk seemed to be limited to subjects with a normal BMI $\left(P_{\text {heterogeneity }}=0.03\right)$ and those who consumed $15 \mathrm{~g}$ or more alcohol per $\mathrm{d}\left(P_{\text {heterogeneity }}=\right.$ 0.02). Associations were similar across strata of age at baseline, level of non-occupational physical activity, lung cancer family history and duration of follow-up. In addition, exclusion of cases diagnosed during the first 2 years of follow-up did not result in noteworthy changes of the effect estimates.

Rule-out approach sensitivity analyses showed that an unmeasured confounder, which increases lung cancer risk three times, 
Table 2. Sex-specific associations of alternate Mediterranean diet score (aMED) and modified Mediterranean diet score (mMED) (in- and excluding the alcohol component) with total lung cancer risk (Hazard ratios (HR) and $95 \%$ confidence intervals)

\begin{tabular}{|c|c|c|c|c|c|c|c|c|c|c|c|c|c|c|c|c|c|c|}
\hline & \multicolumn{9}{|c|}{ aMED in men } & \multicolumn{9}{|c|}{ aMED in women } \\
\hline & \multirow{2}{*}{$\frac{0-3}{\text { Reference }}$} & \multicolumn{2}{|r|}{$4-5$} & \multicolumn{2}{|r|}{$6-8^{*}$} & \multirow[b]{2}{*}{$P_{\text {trend }}$} & \multirow[b]{2}{*}{ AIC } & \multicolumn{2}{|c|}{ Continuous, per 2 pts } & \multirow{2}{*}{$\frac{0-3}{\text { Reference }}$} & \multicolumn{2}{|r|}{$4-5$} & \multicolumn{2}{|r|}{$6-8^{*}$} & \multirow[b]{2}{*}{$P_{\text {trend }}$} & \multirow[b]{2}{*}{ AIC } & \multicolumn{2}{|c|}{ Continuous, per 2 pts } \\
\hline & & $\mathrm{HR}$ & $95 \% \mathrm{Cl}$ & $\mathrm{HR}$ & $95 \% \mathrm{Cl}$ & & & $\mathrm{HR}$ & $95 \% \mathrm{Cl}$ & & $\mathrm{HR}$ & $95 \% \mathrm{Cl}$ & $\mathrm{HR}$ & $95 \% \mathrm{Cl}$ & & & $\mathrm{HR}$ & $95 \% \mathrm{Cl}$ \\
\hline \multicolumn{19}{|l|}{ Excluding alcohol } \\
\hline $\mathrm{PY} / n$ cases & $11842 / 1153$ & & $579 / 970$ & & 784/290 & & & & $05 / 2413$ & $12244 / 209$ & & $116 / 187$ & & $279 / 52$ & & & & $39 / 448$ \\
\hline Age† & 1.00 & 0.80 & $0.69,0.91$ & 0.61 & $0.50,0.74$ & $<0.001$ & & 0.81 & $0.75,0.88$ & 1.00 & 0.72 & $0.57,0.90$ & 0.47 & $0.34,0.66$ & $<0.001$ & & 0.72 & $0.63,0.83$ \\
\hline Smoking $\ddagger$ & 1.00 & 0.84 & $0.72,0.99$ & 0.91 & $0.73,1.14$ & 0.109 & & 0.94 & $0.86,1.04$ & 1.00 & 0.84 & $0.64,1 \cdot 10$ & 0.72 & $0.49,1.06$ & 0.070 & & 0.91 & $0.77,1.06$ \\
\hline Fully adjusted§ & 1.00 & 0.86 & $0.73,1.02$ & 0.91 & $0.72,1.15$ & 0.157 & 33062 & 0.95 & $0.86,1.06$ & 1.00 & 0.87 & $0.65,1.15$ & 0.73 & $0.49,1.09$ & 0.112 & 6006 & 0.91 & $0.77,1.08$ \\
\hline \multirow{6}{*}{$\begin{array}{l}\text { Including alcohol } \\
\text { PY/n cases } \\
\text { Fully adjusted§§| }\end{array}$} & & & & & & & & & & & & & & & & & & \\
\hline & $9196 / 929$ & & 026/1019 & & $983 / 465$ & & & & $05 / 2413$ & $10723 / 172$ & & $688 / 202$ & & 228/74 & & & & $39 / 448$ \\
\hline & 1.00 & 0.86 & $0.72,1.03$ & 0.89 & $0.72,1 \cdot 10$ & 0.177 & 33083 & 0.96 & $0.87,1.05$ & 1.00 & 1.03 & $0.77,1.39$ & 0.80 & $0.55,1.15$ & 0.326 & 6012 & 0.93 & $0.79,1.08$ \\
\hline & \multicolumn{9}{|c|}{ mMED in men } & \multicolumn{9}{|c|}{ mMED in women } \\
\hline & $0-3$ & & $4-5$ & & $6-8^{*}$ & & & Contin & us, per 2 pts & $0-3$ & & $4-5$ & & $6-8^{*}$ & & & Contint & us, per 2 pts \\
\hline & Reference & HR & $95 \% \mathrm{Cl}$ & HR & $95 \% \mathrm{Cl}$ & $P_{\text {trend }}$ & AIC & $\mathrm{HR}$ & $95 \% \mathrm{Cl}$ & Reference & $\mathrm{HR}$ & $95 \% \mathrm{Cl}$ & $\mathrm{HR}$ & $95 \% \mathrm{Cl}$ & $P_{\text {trend }}$ & AIC & $\mathrm{HR}$ & $95 \% \mathrm{Cl}$ \\
\hline \multicolumn{19}{|l|}{ Excluding alcohol } \\
\hline $\mathrm{PY} / n$ cases & $10876 / 941$ & & $539 / 1131$ & & $791 / 341$ & & & & $05 / 2413$ & $11653 / 188$ & & $514 / 207$ & & $473 / 53$ & & & & $39 / 448$ \\
\hline Age† & 1.00 & 0.94 & $0.82,1.08$ & 0.80 & $0.66,0.97$ & 0.031 & & 0.90 & $0.83,0.98$ & 1.00 & 0.77 & $0.62,0.96$ & 0.59 & $0.42,0.82$ & 0.001 & & 0.79 & $0.68,0.90$ \\
\hline Smoking $\ddagger$ & 1.00 & $1 \cdot 10$ & $0.93,1.30$ & 0.99 & $0.79,1.24$ & 0.756 & & 1.01 & $0.91,1.11$ & 1.00 & 0.94 & $0.72,1.24$ & 0.83 & $0.57,1.22$ & 0.382 & & 0.92 & $0.78,1.09$ \\
\hline Fully adjusted§ & 1.00 & $1 \cdot 11$ & $0.93,1.32$ & 0.96 & $0.76,1.21$ & 0.901 & 33066 & 1.00 & $0.90,1.11$ & 1.00 & 0.99 & $0.75,1.32$ & 0.83 & $0.56,1.24$ & 0.474 & 6009 & 0.94 & $0.79,1 \cdot 11$ \\
\hline \multicolumn{19}{|l|}{ Including alcohol } \\
\hline $\mathrm{PY} / n$ cases & $7768 / 679$ & & $071 / 1156$ & & 365/578 & & & & $05 / 2413$ & $9671 / 166$ & & $582 / 198$ & & $386 / 84$ & & & & $39 / 448$ \\
\hline Fully adjusted§̧॥ & 1.00 & 0.99 & $0.82,1.19$ & 0.98 & $0.78,1.21$ & 0.823 & 33094 & 1.02 & $0.92,1.12$ & 1.00 & 0.83 & $0.61,1.11$ & 0.87 & $0.61,1.25$ & 0.339 & 6012 & 0.94 & $0.80,1.11$ \\
\hline
\end{tabular}

AlC, Akaike's information criterion; pts, points; PY, person-years in the subcohort

* The highest score category of the aMED/mMED including the alcohol component was defined as 6-9 points.

† The age-adjusted model was adjusted for age at baseline (years).

¥ The smoking-adjusted model was additionally adjusted for cigarette smoking status (never, former, current), cigarette smoking duration (years, centred) and cigarette smoking frequency (cigarettes smoked per day, centred).

$\S$ The fully adjusted model contained in addition to $¥$ daily energy intake ( $\mathrm{kJ}(\mathrm{kcal}))$, alcohol consumption $(0,>0-<5, \geq 5-<15, \geq 15-<30, \geq 30 \mathrm{~g} / \mathrm{d}), \mathrm{BMI}\left(<18.5, \geq 18.5-<25, \geq 25-<30, \geq 30 \mathrm{~kg} / \mathrm{m}^{2}\right)$, non-occupational physical activity $(\leq 30,>30-\leq 60,>60-\leq 90,>90 \mathrm{~min} / \mathrm{d})$, highest level of education (primary school or lower vocational, secondary school or medium vocational, higher vocational or university), family history of lung cancer (no, yes) and history of physician-diagnosed chronic bronchitis (no, yes).

॥ Not adjusted for alcohol consumption. 


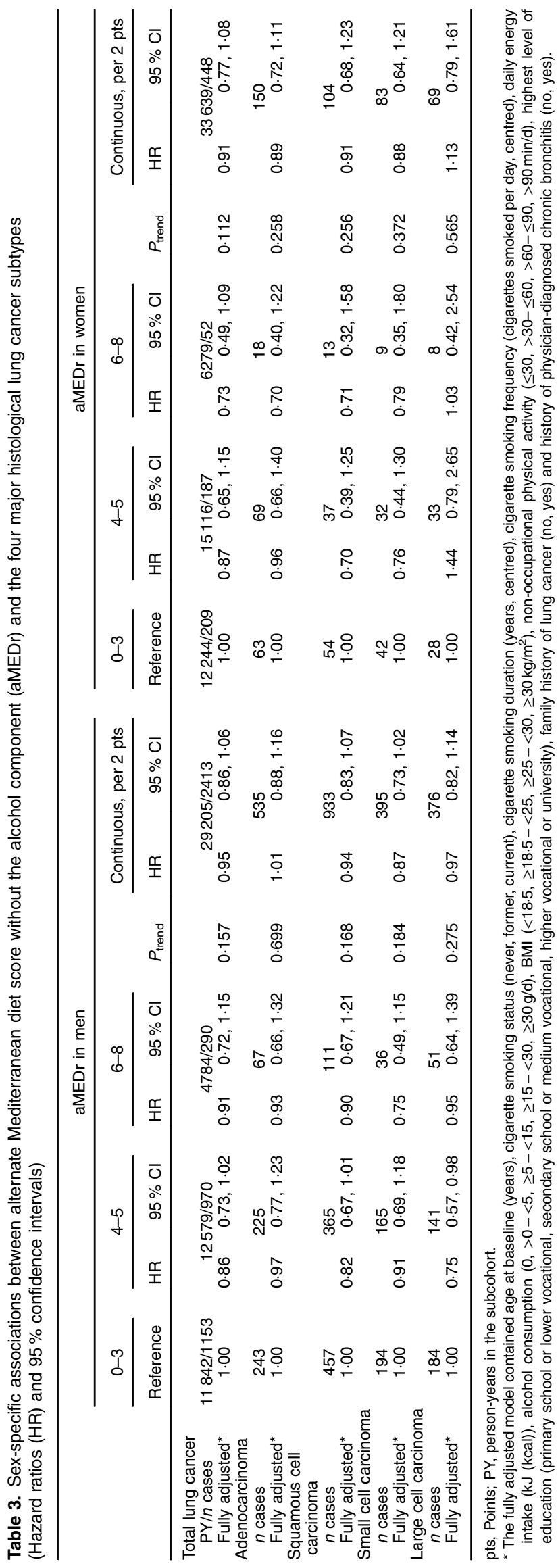

would need to be 3.3 and 2.5 times more common in women and men with low MD adherence, respectively, to be able to explain the observed HR (online Supplementary Fig. S1). A population prevalence of $10 \%$ for the confounder was assumed to obtain these results.

The contribution of the individual aMEDr components to the observed HR was evaluated and results are listed in Table 4. After exclusion of nuts from the score, the inverse association completely disappeared in men. Furthermore, a fully adjusted model including all aMEDr components as dichotomous variables showed that nut intake was statistically significantly associated with a reduced lung cancer risk in men (data not shown). Intakes of fruits and vegetables also considerably contributed to the inverse relation in men. Removal of one of these components weakened the strength of the relation by almost $50 \%$. The inverse association in men gained strength when any of the other components was excluded from the score. In women, intakes of fruits and whole grains, and to a lesser extent the MUFA:SFA ratio, were most strongly associated with a reduced lung cancer risk.

We also compared performances of the relative aMED indices and the absolute WCRF/AICR scores (Table 5). HR were estimated per SD increment to ensure comparability. Both scores showed inverse associations (mostly not statistically significant) with lung cancer risk in men and women, independently of whether alcohol consumption was taken into account in the sum score. HR for the WCRF/AICR score without alcohol were 0.96 (95\% CI 0.88, 1.04) and 0.92 (95\% CI 0.80, 1.07) in men and women, respectively. When the WCRF/AICR score including alcohol was considered, we observed a statistically significant inverse association with lung cancer risk in men (HR per SD increment 0.90; 95\% CI 0.83, 0.98), but not women. The similar AIC values indicated comparable fits of models containing WCRF/AICR score and aMED variables without alcohol in both sexes.

\section{Discussion}

Higher MD adherence was associated with a non-significantly reduced lung cancer risk in the NLCS. HR for comparisons of high to low aMEDr values were 0.73 (95\% CI $0.49,1.09)$ in women and 0.91 (95\% CI $0.72,1.15)$ in men. Associations seemed to vary across the histological subtypes, though heterogeneity tests were not statistically significant. A borderline statistically significant inverse trend was observed in never smokers when the sexes were combined. In our study population, the performance of aMED was superior to that of mMED and better model fits were obtained when alcohol intake was not included in the score.

The results of the present study were in line with those of previous prospective cohort studies ${ }^{(11-13)}$. Nonetheless, in contrast to these studies, the fully adjusted inverse associations observed in the present study lacked statistical significance. In the National Institutes of Health-American Association of Retired Persons (NIHAARP) Diet and Health Study, a HR of 0.85 (95\% CI 0.79, 0.91) for lung cancer was observed when comparing the highest to the lowest aMED quintile ${ }^{(12)}$. An Australian study estimated a HR of 0.64 (95\% CI 0.45, 0.90) for high compared with low MD 
Table 4. Sex-specific associations between alternate Mediterranean diet score without the alcohol component (aMEDr) and total lung cancer risk after alternate exclusion of the individual aMEDr components from the sum score

(Hazard ratios (HR) and $95 \%$ confidence intervals)

\begin{tabular}{|c|c|c|c|c|c|c|c|c|}
\hline \multirow[b]{3}{*}{ aMEDr variants } & \multicolumn{4}{|c|}{ Men } & \multicolumn{4}{|c|}{ Women } \\
\hline & \multirow[b]{2}{*}{ Median intake $(\mathrm{g} / \mathrm{d})^{\star}$} & \multicolumn{2}{|c|}{ Per 2 pts } & \multirow[b]{2}{*}{ HR reduction (\%) } & \multirow[b]{2}{*}{ Median intake $(\mathrm{g} / \mathrm{d})^{*}$} & \multicolumn{2}{|c|}{ Per 2 pts } & \multirow[b]{2}{*}{ HR reduction (\%) } \\
\hline & & $\mathrm{HR} \dagger$ & $95 \% \mathrm{Cl}$ & & & $\mathrm{HR} \dagger$ & $95 \% \mathrm{Cl}$ & \\
\hline Original & & 0.953 & $0.861,1.055$ & & & 0.914 & $0.774,1.079$ & \\
\hline Excluding vegetables & $207 \cdot 3$ & 0.976 & $0.886,1.075$ & 48.9 & $219 \cdot 2$ & 0.908 & $0.763,1.081$ & $-7 \cdot 0$ \\
\hline Excluding legumes & 6.5 & 0.939 & $0.850,1.037$ & -29.8 & 4.7 & 0.915 & $0.778,1.076$ & 1.2 \\
\hline Excluding fruits & $157 \cdot 0$ & 0.976 & $0.886,1.075$ & 48.9 & $209 \cdot 4$ & 0.953 & $0.812,1.119$ & $45 \cdot 3$ \\
\hline Excluding nuts & 3.3 & 0.998 & $0.902,1.105$ & $95 \cdot 7$ & 1.7 & 0.925 & $0.791,1.082$ & $12 \cdot 8$ \\
\hline Excluding whole grains & 0.0 & 0.947 & $0.857,1.046$ & $-12 \cdot 8$ & 0.0 & 0.941 & $0.798,1.111$ & 31.4 \\
\hline $\begin{array}{l}\text { Excluding red and } \\
\text { processed meats }\end{array}$ & $125 \cdot 1$ & 0.950 & $0.863,1.046$ & $-6 \cdot 4$ & $106 \cdot 4$ & 0.926 & $0.793,1.081$ & $14 \cdot 0$ \\
\hline Excluding fish & 11.5 & 0.951 & $0.861,1.051$ & $-4 \cdot 3$ & $8 \cdot 7$ & 0.876 & $0.741,1.035$ & $-44 \cdot 2$ \\
\hline $\begin{array}{l}\text { Excluding MUFA:SFA } \\
\text { ratio }\end{array}$ & 0.98 & 0.933 & $0.846,1.029$ & $-42 \cdot 6$ & 0.94 & 0.930 & $0.796,1.087$ & $18 \cdot 6$ \\
\hline
\end{tabular}

pts, Points; HR reduction, the reduction of the inverse association after exclusion of the respective component from the sum score.

${ }^{*}$ Median intakes of eligible subcohort members are displayed for each of the aMEDr components that were alternately excluded from the sum score.

† HR were adjusted for age at baseline (years), cigarette smoking status (never, former, current), cigarette smoking duration (years, centred), cigarette smoking frequency (cigarettes smoked per day, centred), daily energy intake ( $\mathrm{kJ}(\mathrm{kcal})$ ), alcohol consumption $(0,>0-<5, \geq 5-<15, \geq 15-<30, \geq 30 \mathrm{~g} / \mathrm{d}), \mathrm{BMI}(<18.5, \geq 18.5-<25, \geq 25-<30$, $\left.\geq 30 \mathrm{~kg} / \mathrm{m}^{2}\right)$, non-occupational physical activity $(\leq 30,>30-\leq 60,>60-\leq 90,>90 \mathrm{~min} / \mathrm{d})$, highest level of education (primary school or lower vocational, secondary school or medium vocational, higher vocational or university), family history of lung cancer (no, yes), history of physician-diagnosed chronic bronchitis (no, yes) and the excluded aMEDr component (<median, $\geq$ median).

Table 5. Sex-specific associations of the absolute World Cancer Research Fund/American Institute for Cancer Research (WCRF/AICR) score and alternate Mediterranean diet score (aMED) with total lung cancer risk

(Hazard ratios (HR) and $95 \%$ confidence intervals)

\begin{tabular}{|c|c|c|c|c|c|c|}
\hline & \multicolumn{3}{|c|}{ Men } & \multicolumn{3}{|c|}{ Women } \\
\hline & \multicolumn{2}{|c|}{ Per SD* } & \multirow[b]{2}{*}{ AIC } & \multicolumn{2}{|c|}{ Per SD* } & \multirow[b]{2}{*}{$\mathrm{AIC}$} \\
\hline & $\mathrm{HR} \dagger$ & $95 \% \mathrm{Cl}$ & & $\mathrm{HR} \dagger$ & $95 \% \mathrm{Cl}$ & \\
\hline $\mathrm{PY} / n$ cases & $28564 / 2341 \ddagger$ & & & 32 992/441‡ & & \\
\hline \multicolumn{7}{|l|}{ Excluding alcohol } \\
\hline WCRF/AICR score§ & 0.96 & $0.88,1.04$ & 31960 & 0.92 & $0.80,1.07$ & 5895 \\
\hline aMEDr & 0.96 & $0.89,1.05$ & 31961 & 0.94 & $0.83,1.08$ & 5896 \\
\hline \multicolumn{7}{|l|}{ Including alcohol } \\
\hline WCRF/AICR score§॥ & 0.90 & $0.83,0.98$ & 31960 & 0.94 & $0.81,1.08$ & 5902 \\
\hline aMED\| & 0.97 & $0.89,1.05$ & 31980 & 0.94 & $0.83,1.07$ & 5902 \\
\hline
\end{tabular}

AIC, Akaike's information criterion; PY, person-years in the subcohort; aMEDr, alternate Mediterranean diet score without the alcohol component.

${ }^{*} \mathrm{HR}$ were estimated per sex-specific SD increment in the scores.

†HR were adjusted for age at baseline (years), cigarette smoking status (never, former, current), cigarette smoking duration (years, centred), cigarette smoking frequency (cigarettes smoked per day, centred), daily energy intake (kJ (kcal)), alcohol consumption $(0,>0-<5, \geq 5-<15, \geq 15-<30, \geq 30 \mathrm{~g} / \mathrm{d}), \mathrm{BMI}(<18.5, \geq 18.5-<25, \geq 25-<30$, $\left.\geq 30 \mathrm{~kg} / \mathrm{m}^{2}\right)$, non-occupational physical activity $(\leq 30,>30-\leq 60,>60-\leq 90,>90 \mathrm{~min} / \mathrm{d}$ ), highest level of education (primary school or lower vocational, secondary school or medium vocational, higher vocational or university), family history of lung cancer (no, yes) and history of physician-diagnosed chronic bronchitis (no, yes).

$\ddagger$ A lower number of subjects could be included in these analyses as a result of missing values for salt intake.

$\S$ Score based on WCRF/AICR dietary recommendations to prevent cancer.

II Not adjusted for alcohol consumption.

adherence, based on an adapted version of tMED ${ }^{(13)}$. Similarly, an inverse association was found in an Italian study among (former) heavy smokers ${ }^{(11)}$. HR varied between 0.58 and 0.66 when comparing aMED values of $2-3,4-5$ and $6-7$ to a value of $0-1$. A substantial reduction in lung cancer risk (90\%) was associated with having an aMED value of 8-9, but this was only based on one lung cancer case.

When we combined men and women, we found a HR of 0.89 (95\% CI $0.73,1.10$ ) for high $v$. low MD adherence (aMEDr). Compared with our study, the inverse associations observed in previously conducted cohort studies were stronger ${ }^{(11-13)}$. This difference might be attributed to higher proportions of Mediterranean subjects, particularly in the Australian study ${ }^{(13,40)}$, which deliberately oversampled Italian and Greek migrants (approximately one-quarter of the cohort), and the Italian study ${ }^{(11)}$, possibly resulting in larger contrasts in absolute food intakes between subjects with high and low MD adherence. Furthermore, wide intake distributions were noted in the NIH-AARP cohort $^{(41)}$. Methods used to adjust for cigarette smoking in the NIH-AARP study and the Australian study seemed to be less comprehensive compared with the method used in the present study, increasing the likelihood that residual confounding by cigarette smoking could have impacted the results of these studies. The NIH-AARP cohort did not take the duration of smoking into account ${ }^{(12)}$, whereas smoking duration and frequency were combined into the composite measure 
'pack-years' in the Australian study ${ }^{(13)}$. Nevertheless, nonsignificant inverse associations between MD adherence and lung cancer risk were present in never smokers in both studies, which is similar to observations in male and female NLCS participants who never smoked. In addition, in never smoking NLCS participants, non-significant inverse associations were observed with all histological subtypes. Therefore, we consider a large impact of residual confounding by cigarette smoking on the results of our study unlikely.

Sex-specific associations of MD adherence with lung cancer risk have seldom been reported in prior studies. Only the NIHAARP study showed sex-specific associations and reported a statistically significant inverse relation between MD adherence and lung cancer risk in both sexes ${ }^{(12)}$. In our study, MD adherence seemed to have a slightly stronger inverse relation with lung cancer risk in women compared with men. Because this heterogeneity was not statistically significant and data from previous research is mostly lacking, additional research on sex-specific associations between MD adherence and lung cancer risk is required before any conclusions can be drawn regarding this topic.

The association of MD adherence with the histological lung cancer subtypes has rarely been evaluated as well. Higher MD adherence was statistically significantly associated with a reduced risk of adenocarcinoma and squamous cell carcinoma, but not small cell carcinoma, in the NIH-AARP cohort $^{(12)}$. Although the heterogeneity tests were not statistically significant, results of our study suggested that associations with MD adherence might vary across the lung cancer subtypes, particularly in men. However, the currently available evidence is not sufficient to conclude anything concerning possible variations in associations of the lung cancer subtypes with MD adherence.

Results of the rule-out approach sensitivity analyses suggested that a strong unmeasured lung cancer risk factor with appreciable inequalities in its distribution over the MD adherence categories would be required to fully explain the observed associations. Occupational exposure to asbestos, for instance, substantially increases lung cancer risk ${ }^{(42)}$. However, it is questionable whether its association with $\mathrm{MD}$ adherence is sufficiently strong and we did adjust for level of education. Other lung cancer risk factors that remained unmeasured were assumed to generally be weak. So, it is unlikely that unmeasured confounders have substantially impacted our study results.

A previous cohort study showed that, of the components included in aMEDr, whole grains and fruits had the strongest inverse relation with both male and female lung cancer risk ${ }^{(12)}$. We reported similar results with respect to female lung cancer risk. However, nut intake seemed to be the most important driver of the inverse association observed in male NLCS participants. Some individual components were not associated with lung cancer risk or had associations in unexpected directions, emphasising the potential advantages of analysis of dietary patterns as opposed to individual dietary components. Dietary pattern analysis takes into account that individual dietary components may interact synergistically or antagonistically while exerting their effects on health. In addition, weak and otherwise undetectable effects of singular components may become detectable if they are combined in dietary patterns. Furthermore, foods are generally consumed in certain patterns, raising collinearity and confounding issues when individual components are evaluated. Finally, by grouping dietary components together, contrasts in healthiness of the diet within the study population will probably increase, thereby increasing the chance of detecting true effects, if present ${ }^{(23,43,44)}$.

Evidence on the relation between alcohol consumption and lung cancer risk is inconclusive ${ }^{(14)}$. Therefore, we excluded alcohol from the MD scores and evaluated the effect of this exclusion. Both the present and a previous NLCS analysis showed better performances of models using MD scores without $_{\text {alcohol }}^{(37)}$. However, earlier cohort studies regarding MD adherence and lung cancer risk all considered moderate alcohol consumption as a beneficial component of the MD score used. Similar to a previous NLCS analysis concerning breast cancer, model performances were better when aMED variants were included compared with mMED variants ${ }^{(37)}$. aMED variants consider whole grains as a beneficial component, whereas whole grains and refined grains are combined in mMED variants, despite their potentially differential health effects ${ }^{(45)}$. Similarly, mMED variants include total meat consumption, whereas aMED variants consider the intake of red and processed meats, specifically ${ }^{(11,46-48)}$. These and other differences in the composition of the food components included might explain the better performance of aMED variants in our study population.

Reduced levels of reactive oxygen species-induced DNA damage, oxidative stress, and inflammation may mediate the potential cancer-protective effect of the $\mathrm{MD}^{(44,49)}$. Consumption of vegetables, legumes, fruits, nuts, whole grains and olive oil is high in the plant-based $\mathrm{MD}^{(5,6)}$. These foods are rich in dietary fibre, vitamins, minerals and polyphenols and have been associated with high levels of antioxidants and low levels of oxidised LDL-cholesterol $^{(44,49-54)}$. Furthermore, polyphenols may exert anti-inflammatory effects ${ }^{(55)}$.

It is a potential weakness that the interpretation of the value of aMEDr depends on the study population, particularly in a non-Mediterranean population. Therefore, we compared the performance of this score to that of an absolute WCRF/AICR score. Generally, the performance of the aMEDr and the WCRF/ AICR score without alcohol was comparable. The WCRF/AICR score without alcohol showed a non-significant and weak inverse association with lung cancer risk in both sexes. A SD increment in WCRF/AICR score including alcohol was statistically significantly associated with a reduced lung cancer risk in men, but not women. Higher adherence to the WCRF/AICR recommendations has previously been associated with a reduced lung cancer risk in the EPIC cohort (HR per one-point increment $0.92 ; 95 \%$ CI $0.89,0.96)^{(38)}$. In contrast, a WCRF/ AICR score was not associated with lung cancer risk in the elderly according to a meta-analysis within the CHANCES consortium (HR per one-point increment 0.99; 95\% CI 0.84, $1 \cdot 17)^{(56)}$, indicating that additional research is warranted.

The NLCS cohort has a high follow-up completion rate, which minimised the risk of information and selection bias. In addition, the large number of lung cancer cases allowed extensive 
adjustment for potential confounding, subtype-specific analyses and analyses stratified by smoking status. A potential weakness of the NLCS is the single assessment of dietary habits and potential confounders at baseline, which may have resulted in attenuated associations. Also, measurement errors in the assessment of dietary intake cannot fully be excluded and might have resulted in misclassification. The dependence of aMEDr on the study population is a final potential weakness. Because our study was conducted in a non-Mediterranean population, one may question the extent to which high values of aMEDr reflected a truly Mediterranean way of eating.

Though the inverse associations lacked statistical significance, the present study is in agreement with other cohort studies showing that high MD adherence might be associated with a reduced lung cancer risk and suggested that this inverse association might also be generalisable to non-Mediterranean populations. In addition, we observed potential differences in associations between the sexes and histological subtypes warranting future research. Finally, exclusion of alcohol from MD scores should be investigated more extensively, primarily with respect to a potential role of the MD in cancer prevention.

\section{Acknowledgements}

The authors would like to thank the participants of the Netherlands Cohort Study (NLCS), the Netherlands Cancer Registry and the Dutch Pathology Registry. Furthermore, NLCS staff members are acknowledged for their valuable assistance and advice.

This study was supported by the Wereld Kanker Onderzoek Fonds Nederland (WCRF-NL), as part of the World Cancer Research Fund International grant programme (grant no. 2015/ 1390). The WCRF-NL had no role in the design, analysis or writing of this article.

Both authors have made large contributions to the work described in the current paper. M. S. was involved in the formulation of the research questions, design of the research, data analysis, interpretation of the results and writing of the manuscript. P. A. v. d. B. was involved in the initiation and coordination of the NLCS, the formulation of the research questions, design of the research, collection of the data, coordination of the analyses, interpretation of the results and critically reviewed the manuscript.

The authors declare that there are no conflicts of interest.

\section{Supplementary material}

For supplementary material/s referred to in this article, please visit https://doi.org/10.1017/S0007114517003737

\section{References}

1. Ferlay J, Soerjomataram I, Ervik M, et al. (2013) GLOBOCAN 2012 v1.0, Cancer Incidence and Mortality Worldwide: IARC CancerBase No. 11. Lyon: International Agency for Research on Cancer. http://globocan.iarc.fr (accessed July 2016).

2. Howlader $\mathrm{N}$, Noone $\mathrm{AM}$, Krapcho $\mathrm{M}$, et al. (2016) SEER Cancer Statistics Review, 1975-2013. Bethesda, MD: National Cancer Institute. http://seer.cancer.gov/csr/1975_2013/ based on November 2015 SEER data submission, posted to the SEER web site, April 2016 (accessed February 2017).

3. Integraal Kankercentrum Nederland (2017) Nederlandse Kankerregistratie. http://www.cijfersoverkanker.nl/ (accessed February 2017).

4. World Cancer Research Fund \& American Institute for Cancer Research (2007) Food, Nutrition, Physical Activity, and the Prevention of Cancer: A Global Perspective. Washington, DC: American Institute for Cancer Research.

5. Willett WC, Sacks F, Trichopoulou A, et al. (1995) Mediterranean diet pyramid: a cultural model for healthy eating. $A m J$ Clin Nutr 61, 1402S-1406S.

6. Trichopoulou A \& Lagiou P (1997) Healthy traditional Mediterranean diet: an expression of culture, history, and lifestyle. Nutr Rev 55, 383-389.

7. Fung TT, Rexrode KM, Mantzoros CS, et al. (2009) Mediterranean diet and incidence of and mortality from coronary heart disease and stroke in women. Circulation 119, 1093-1100.

8. Sofi F, Macchi C, Abbate R, et al. (2014) Mediterranean diet and health status: an updated meta-analysis and a proposal for a literature-based adherence score. Public Health Nutr 17 , $2769-2782$

9. Martinez-Gonzalez MA \& Bes-Rastrollo M (2014) Dietary patterns, Mediterranean diet, and cardiovascular disease. Curr Opin Lipidol 25, 20-26.

10. Grosso G, Marventano S, Yang J, et al. (2017) A comprehensive meta-analysis on evidence of Mediterranean diet and cardiovascular disease: are individual components equal? Crit Rev Food Sci Nutr 57, 3218-3232.

11. Gnagnarella P, Maisonneuve P, Bellomi M, et al. (2013) Red meat, Mediterranean diet and lung cancer risk among heavy smokers in the COSMOS screening study. Ann Oncol 24, 2606-2611.

12. Anic GM, Park Y, Subar AF, et al. (2016) Index-based dietary patterns and risk of lung cancer in the NIH-AARP Diet and Health Study. Eur J Clin Nutr 70, 123-129.

13. Hodge AM, Bassett JK, Shivappa N, et al. (2016) Dietary inflammatory index, Mediterranean diet score, and lung cancer: a prospective study. Cancer Causes Control 27, 907-917.

14. International Agency for Research on Cancer (2010) IARC Monographs on the Evaluation of Carcinogenic Risks to Humans: Alcohol Consumption and Ethyl Carbamate, vol. 96. Lyon: International Agency for Research on Cancer.

15. van den Brandt PA, Goldbohm RA, van 't Veer P, et al. (1990) A large-scale prospective cohort study on diet and cancer in The Netherlands. J Clin Epidemiol 43, 285-295.

16. van den Brandt PA, Schouten LJ, Goldbohm RA, et al. (1990) Development of a record linkage protocol for use in the Dutch Cancer Registry for Epidemiological Research. Int J Epidemiol 19, 553-558.

17. Goldbohm RA, van den Brandt PA, Brants HA, et al. (1994) Validation of a dietary questionnaire used in a large-scale prospective cohort study on diet and cancer. Eur J Clin Nutr 48, 253-265.

18. Volovics A \& van den Brandt PA (1997) Methods for the analyses of case-cohort studies. Biometrical J 39, 195-214.

19. van den Brandt PA, van't Veer P, Goldbohm RA, et al. (1993) A prospective cohort study on dietary fat and the risk of postmenopausal breast cancer. Cancer Res 53, 75-82.

20. Goldbohm RA, van 't Veer P, van den Brandt PA, et al. (1995) Reproducibility of a food frequency questionnaire and stability of dietary habits determined from five annually repeated measurements. Eur J Clin Nutr 49, 420-429.

21. NEVO table (1986) Dutch Food Composition Table 1986-1987. The Hague: Voorlichtingsbureau voor de Voeding. 
22. Trichopoulou A, Kouris-Blazos A, Wahlqvist ML, et al. (1995) Diet and overall survival in elderly people. BMJ 311, $1457-1460$.

23. Trichopoulou A, Costacou T, Bamia C, et al. (2003) Adherence to a Mediterranean diet and survival in a Greek population. N Engl J Med 348, 2599-2608.

24. Trichopoulou A, Orfanos P, Norat T, et al. (2005) Modified Mediterranean diet and survival: EPIC-elderly prospective cohort study. BMJ 330, 991.

25. Fung TT, McCullough ML, Newby PK, et al. (2005) Dietquality scores and plasma concentrations of markers of inflammation and endothelial dysfunction. Am J Clin Nutr $\mathbf{8 2}$, $163-173$.

26. Mitrou PN, Kipnis V, Thiebaut AC, et al. (2007) Mediterranean dietary pattern and prediction of all-cause mortality in a US population: results from the NIH-AARP Diet and Health Study. Arch Intern Med 167, 2461-2468.

27. Lin DY \& Wei LJ (1989) The Robust Inference for the Cox Proportional Hazards Model. J Am Stat Assoc 84, 1074-1078.

28. Grambsch PM \& Therneau TM (1994) Proportional hazards tests and diagnostics based on weighted residuals. Biometrika 81, 515-526.

29. Akaike H (1974) A new look at the statistical model identification. IEEE Trans Autom Control AC-19, 716-723.

30. Rockhill B, Newman B \& Weinberg C (1998) Use and misuse of population attributable fractions. Am J Public Health 88, 15-19.

31. Newson RB (2013) Attributable and unattributable risks and fractions and other scenario comparisons. Stata $J \mathbf{1 3}$, 672-698.

32. Wahrendorf J (1987) An estimate of the proportion of colorectal and stomach cancers which might be prevented by certain changes in dietary habits. Int J Cancer 40, 625-628.

33. Wacholder S, Gail MH, Pee D, et al. (1989) Alternative variance and efficiency calculations for the case-cohort design. Biometrika 76, 117-123.

34. de Vogel S, Bongaerts BW, Wouters KA, et al. (2008) Associations of dietary methyl donor intake with MLH1 promoter hypermethylation and related molecular phenotypes in sporadic colorectal cancer. Carcinogenesis 29, 1765-1773.

35. Schneeweiss S (2006) Sensitivity analysis and external adjustment for unmeasured confounders in epidemiologic database studies of therapeutics. Pharmacoepidemiol Drug Saf 15, 291-303.

36. Trichopoulou A, Bamia C \& Trichopoulos D (2009) Anatomy of health effects of Mediterranean diet: Greek EPIC prospective cohort study. BMJ 338, b2337.

37. van den Brandt PA \& Schulpen M (2017) Mediterranean diet adherence and risk of postmenopausal breast cancer: results of a cohort study and meta-analysis. Int J Cancer 140, 2220-2231.

38. Romaguera D, Vergnaud AC, Peeters PH, et al. (2012) Is concordance with World Cancer Research Fund/American Institute for Cancer Research guidelines for cancer prevention related to subsequent risk of cancer? Results from the EPIC study. Am J Clin Nutr 96, 150-163.

39. Vergnaud AC, Romaguera D, Peeters PH, et al. (2013) Adherence to the World Cancer Research Fund/American Institute for Cancer Research guidelines and risk of death in Europe: results from the European Prospective Investigation into Nutrition and Cancer cohort study. Am J Clin Nutr 97, $1107-1120$.

40. Harriss LR, English DR, Powles J, et al. (2007) Dietary patterns and cardiovascular mortality in the Melbourne Collaborative Cohort Study. Am J Clin Nutr 86, 221-229.

41. Schatzkin A, Subar AF, Thompson FE, et al. (2001) Design and serendipity in establishing a large cohort with wide dietary intake distributions : the National Institutes of HealthAmerican Association of Retired Persons Diet and Health Study. Am J Epidemiol 154, 1119-1125.

42. Ngamwong $\mathrm{Y}$, Tangamornsuksan W, Lohitnavy $\mathrm{O}$, et al. (2015) Additive Synergism between Asbestos and Smoking in Lung Cancer Risk: A Systematic Review and Meta-Analysis. PLoS One 10, e0135798.

43. Jacques PF \& Tucker KL (2001) Are dietary patterns useful for understanding the role of diet in chronic disease? Am J Clin Nutr 73, 1-2.

44. Verberne L, Bach-Faig A, Buckland G, et al. (2010) Association between the Mediterranean diet and cancer risk: a review of observational studies. Nutr Cancer 62, 860-870.

45. Aune D, Chan DS, Lau R, et al. (2011) Dietary fibre, whole grains, and risk of colorectal cancer: systematic review and doseresponse meta-analysis of prospective studies. BMJ 343, d6617.

46. Chan DS, Lau R, Aune D, et al. (2011) Red and processed meat and colorectal cancer incidence: meta-analysis of prospective studies. PLOS ONE 6, e20456.

47. Yang WS, Wong MY, Vogtmann E, et al. (2012) Meat consumption and risk of lung cancer: evidence from observational studies. Ann Oncol 23, 3163-3170.

48. Shi Y, Yu PW \& Zeng DZ (2015) Dose-response meta-analysis of poultry intake and colorectal cancer incidence and mortality. Eur J Nutr 54, 243-250.

49. Brill JB (2009) The Mediterranean diet and your health. Am J Lifestyle Med 3, 44-56.

50. Ferro-Luzzi A \& Branca F (1995) Mediterranean diet, Italian-style: prototype of a healthy diet. Am J Clin Nutr 61, 1338S-1345S.

51. Kushi LH, Lenart EB \& Willett WC (1995) Health implications of Mediterranean diets in light of contemporary knowledge. 1. Plant foods and dairy products. Am J Clin Nutr 61, 1407S-1415S.

52. Marrugat J, Covas MI, Fito M, et al. (2004) Effects of differing phenolic content in dietary olive oils on lipids and LDL oxidation - a randomized controlled trial. Eur J Nutr 43, 140-147.

53. Pitsavos C, Panagiotakos D, Trichopoulou A, et al. (2006) Interaction between Mediterranean diet and methylenetetrahydrofolate reductase $\mathrm{C677T}$ mutation on oxidized low density lipoprotein concentrations: the ATTICA study. Nutr Metab Cardiovasc Dis 16, 91-99.

54. Bogani P \& Visioli F (2007) Antioxidants in the Mediterranean diets: An update. World Rev Nutr Diet 97, 162-179.

55. Rahman I, Biswas SK \& Kirkham PA (2006) Regulation of inflammation and redox signaling by dietary polyphenols. Biochem Pharmacol 72, 1439-1452.

56. Jankovic N, Geelen A, Winkels RM, et al. (2017) Adherence to the WCRF/AICR dietary recommendations for cancer prevention and risk of cancer in elderly from Europe and the United States: a meta-analysis within the CHANCES project. Cancer Epidemiol Biomarkers Prev 26, 136-144. 